\title{
Automatic Diameter and Orientation Distribution Determination of Fibrous Materials in micro X-Ray CT Imaging Data
}

\author{
John P. Chiverton*1, Alexander Kao ${ }^{1}$, Marta Roldo ${ }^{2}$, and Gianluca Tozzi ${ }^{1}$ \\ ${ }^{1}$ School of Engineering, University of Portsmouth, Portsmouth, UK, PO1 3DJ \\ ${ }^{2}$ School of Pharmacy and Institute of Biomedical and Biomolecular Science, \\ University of Portsmouth, UK, PO1 2DT
}

\section{Abstract}

Fibrous nano-materials such as electrospun materials have many uses ranging from tissue engineering to biosensors. High-resolution imaging is an important component in the characterisation of these materials. Important parameters required to predict and study the properties of fibre rich materials include diameter and orientation distribution as well as fibre spacing. The orientations and the relative dimensions of the fibres can be measured via specially designed imaging software. Difficulties in this measurement process can arise if fibres are distributed in close proximity to each other in relation to the resolution of the imaging modality. For example, if some automation is required in the measurement process and, particularly, if the automated processes are not designed for situations where the fibres are in close proximity to each other. This work is therefore concerned with the development of automated measurement techniques to provide estimates of the diameters of fibres and also the orientation distribution. The software automatically detects special points in the fibrous materials where fibres can be considered to have some delineation from surrounding fibres. These sparse points are considered to be points at which estimates of the fibres' properties can be quantified. Aligned and randomly distributed electrospun PCL nanofibres were prepared. Imaging of these materials was performed with an X-Ray Computer Tomography (XCT) system with an image voxel size of $0.15 \times 0.15 \times 0.15 \mu \mathrm{m}^{3}$. Scanning Electron Microscopy (SEM) images were also obtained. Fibre diameters estimated using images from both modalities using the developed techniques were found to be in agreement. Orientation distribution was summarised with multiscale Entropy and found to be consistent with visual observation across different scales.

Keywords: 3D Image Processing, XCT, Fibres, Electrospinning, PCL

\footnotetext{
*Corresponding author: john.chiverton@port.ac.uk
} 

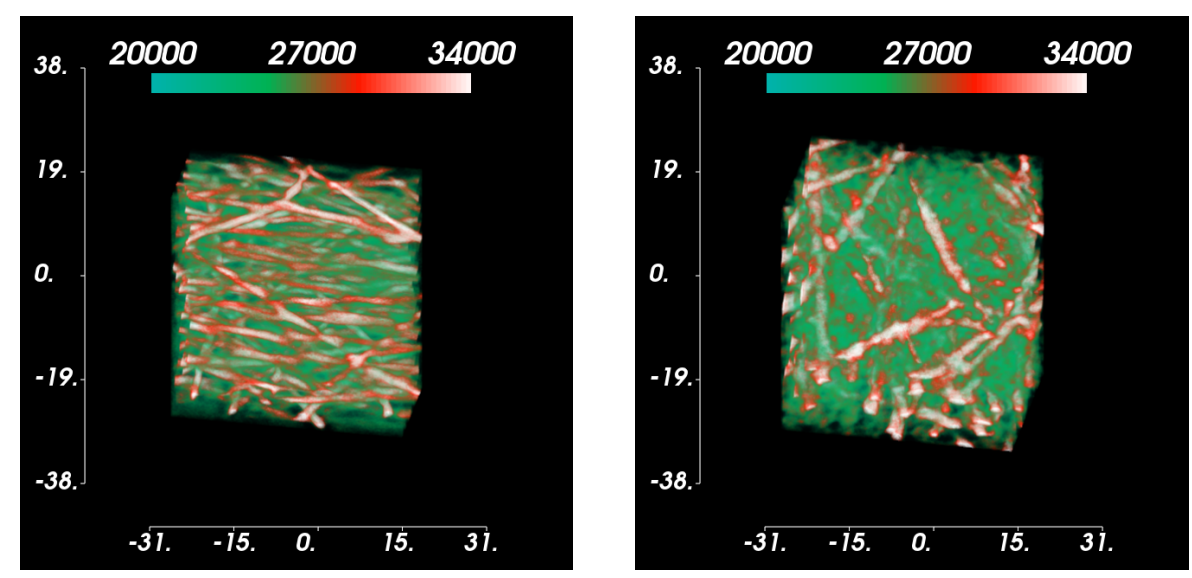

Figure 1: Three dimensional visualization of sub-volumes or regions of interests of a sample with (left) aligned fibres and (right) random fibres. Units along axes are in $\mu \mathrm{m}$.

\section{Introduction}

Fibrous nano-materials are important for many potential applications including: wound dressing (Hassiba et al., 2017); filtration, including air filtration (Al-Attabi et al., 2018); biosensors (Pasinszki et al., 2017); tissue engineering such as skin tissue engineering (Jiang et al., 2017); and targeted drug delivery to e.g. bone (Cheng et al., 2017). Fibres are also naturally occurring in plants, and in animals, see e.g. (Mansfield et al., 2009) who investigated the presence of collagen and elastin in articular cartilage; and (Mostao-Guidolin et al., 2017) who investigated imaging techniques applied to the imaging of collagen fibres in scar tissue.

Fibrous nano-materials can be fabricated using a variety of techniques such as pull spinning, electrospinning and melt spinning, as compared in (Deravi et al., 2017). Electrospinning is considered here because of its general applicability as it can be used to fabricate fibres consisting of polymers or biological materials such as collagen and elastin if desired, such as in (Sensini et al., 2017).

An important consideration in the application of man-made fibrous materials is the characterisation of the fabricated material to determine the suitability and potential efficacy for the intended application. Imaging can be used to acquire images of the material to help in this characterisation process. Possible parameters of interest can include fibre diameters and fibre orientation distribution, see e.g. (Yau et al., 2015). A common imaging modality used to image electrospun fibres is Scanning Electron Microscopy (SEM). SEM is useful as it can provide resolutions down to $10 \mathrm{~nm}$, but it is only able to provide a surface view of the sample of interest non-destructively. Imaging in 3D can be performed with Focused Ion Beam - Scanning Electron Microscopy (FIB-SEM) although FIB-SEM is destructive, by the Focused lon Beam. Another technique, which is not destructive is nano or micro X-ray Computed Tomography (XCT) imaging. Three-dimensional imaging is useful as it can help to characterise the sample throughout, rather than one based on a surface view of the material. Also possibly providing resolutions down to around $100 \mathrm{~nm}$ or less in some instances depending on the system in use.

Volume renderings of example electrospun fibres imaged using XCT can be seen in Fig. 1. The work presented here considers the development of automatic techniques that can help in the characterisation of these materials from imaging data. This can be useful to gain an insight of the material's role in various applications. Fibre diameter and the orientation distribution are parameters that are often estimated using semi-manual interaction with image processing tools such as in (Ahmed et al., 2016; Hotaling et al., 2015). Manual interaction with image processing tools can be highly informative however there can be a possibility with difficulties in repeatability, see e.g. (Sims et al., 2002). 

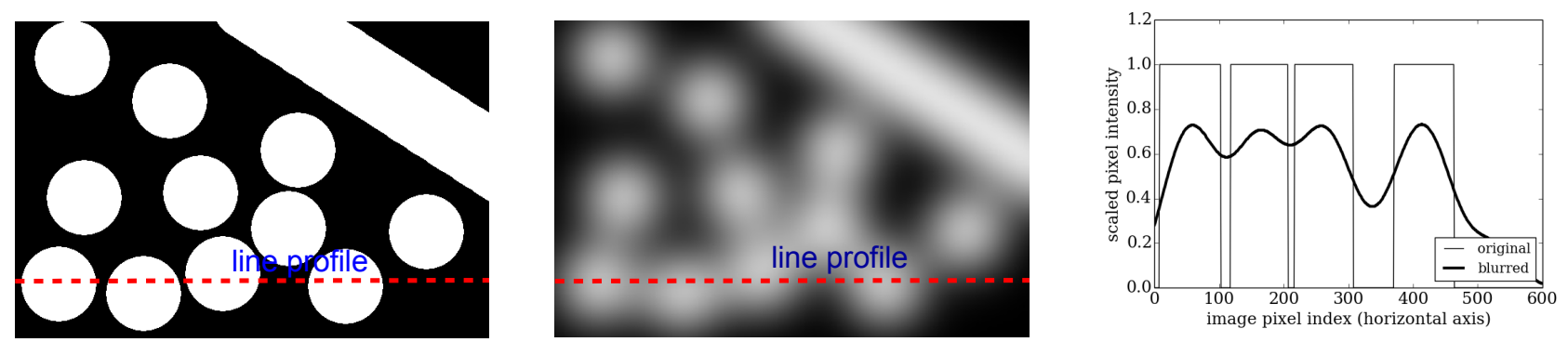

Figure 2: Illustration of the effect of a Point Spread Function (PSF) on a synthetic image example. Left: shows a synthetic image unaffected by the action of the PSF; middle: shows the image after the PSF has been convolved with the image; and right: shows the line profiles for both.

Many techniques also exist to automatically decide the point at which a fibre's boundary begins and ends. This process might, for instance, be considered to require determining optimal thresholds, particularly given a bimodal frequency distribution of image intensities as used in (Chiverton et al., 2017). However if the spread of image intensities are particularly wide then the bimodality can become unimodal where the background may share a similar range of intensities with the foreground. Furthermore direct image thresholding is not directly applicable to Partial Volume (PV) affected imaging data, see e.g. (Chiverton and Wells, 2008). The PV effect is a term used to describe the effect of the blurring of the imaging data that is a result of the convoluting action of the Point Spread Function (PSF) and possibly also any associated resolution limiting effects. The PV effect is illustrated in Fig. 2. The PV effect is an important consideration not often explicitly considered in existing works on fibre segmentation and tracking, particularly in XCT imaging.

Measurements of structures of interest such as the diameters of fibres can therefore require more sophisticated approaches. This is particularly true if the spread of the image intensities is wide in relation to the structure of interest which is often the case with imaging of fibre based materials and tissues. Another point of difficulty can arise when the fibres have close proximity in relation to the spread of the intensities, which again is a likely scenario. Difficulties such as poor interslice sampling can however be dealt with using robust fitting algorithms such as the work by (Chiverton et al., 2017). Imaging artefacts may also result in inhomogeneities in the image intensities as described by (Barrett and Keat, 2004) which may be more of a problem for denser materials.

The PV effect is particularly relevant to applications at a smaller scale. For example, XCT of nano-scale fibres can present some difficulties, partly due to the smaller scale which can require additional modelling of this PV effect. Another consideration could potentially include the difficulty of accurately segmenting fibres that possess similar appearance to the surrounding materials in the imaging data.

For instance, machine learning was used by (Emerson et al., 2017) to detect centre points of fibres in XCT 3D images of glass and carbon fibre composites. The authors demonstrated the difficulties associated with intensity thresholding to detect centre points. Instead Emerson et al. (2017) developed a technique to recognise likely centre points of fibres. Centre points were then interconnected to enable fibre trajectories to be obtained. Another machine learning centric approach that has attracted some interest is the work by Kitrungrotsakul et al. (2017) which used a deep convolution neural network applied to the segmentation of hepatic blood vessels in Computer Tomography (CT) imaging data. The machine learning technique enabled the technique to automatically learn relevant features that characterised the vessels. 
Tubular structures, such as blood vessels have previously been detected using various forms of eigen-analysis of the Hessian of 3D imaging data, see e.g. (Frangi et al., 1998; Sato et al., 1998; Drechsler and Laura, 2010; Rudyanto et al., 2014). This approach was proposed by (Sato et al., 1998) and found repeated use over the years (Drechsler and Laura, 2010; Jimenez-Carretero et al., 2013; Rudyanto et al., 2014). Airways, e.g. (Jimenez-Carretero et al., 2013), have also been similarly modelled. This is the approach taken here, i.e. to model fibres as tubular structures and enhance them through preprocessing at multiple scales using eigen-analysis. This is similar to e.g. (Frangi et al., 1998; Sato et al., 1998) except it is adapted here to work with fibres at small scales, in relation to the imaging resolution, and the fibres are in close proximity.

A different approach, based on fibre tracing, has previously been investigated in e.g. (Rigort et al., 2012; Weber et al., 2012). Fibre tracing is often combined with other techniques such as in conjunction with the use of a distance transform (Huang et al., 2016). Huang et al. investigated techniques applicable to XCT of Porous Metal Fibre Sintered Sheet (PMFSS) material, as used in fuel cells.

Another interesting work is (Maksimcuka et al., 2017) which considered the characteristics of a sample once it had undergone mechanical deformation. Distribution of fibre diameters and the orientation of the fibres were found to be important characteristics in that work. It also used fibre tracing, using correlation to match fibres, based on the works in (Rigort et al., 2012; Weber et al., 2012). However it was not clear whether some manual interaction was required, e.g. at the point to fit ellipses to determine mean fibre diameters although relatively trivial to implement using modern image processing libraries such as the Insight ToolKit (ITK) as described by (Johnson et al., 2013).

Other authors have considered materials consisting of naturally occurring based fibres such as paper and wood based composite material as shown by (Axelsson, 2008; Axelsson and Svensson, 2010). The authors used a structure tensor of derivatives, similar to the Hessian, except the Hessian consists of second derivatives. The authors had to select the scale of the filters according to the size of the structure they were interested.

Automation of these image summarisation techniques is made more difficult when fibres have close proximity and small dimensions, as might be found for electrospun fibres even when using image data acquired with sub-micron voxel sizes. An automated sampling methodology has therefore been developed here that can detect points in the imaging data where fibres are present and which are well separated from neighbouring fibres. These points provide optimal locations to perform orientation and thickness measurements and thus enable automated measurements of these variables. Imaging data of fibrous materials is collected using the Zeiss Xradia Versa 510 Microtomography X-Ray Computer Tomography (XCT) system. Diameter measurements are automatically obtained which match well with the expected thickness of the manufactured material, across a number of scales. The orientation distribution is also calculated, visualised and further summarised using a single parameter measure together with the distribution of the fibres throughout the scanned volume.

\section{Methodology}

The 3D Hessian matrix $\mathcal{H}_{\eta}$ at scale $\eta$ of second derivatives of a 3D image volume $I$ can be computed for every voxel position $\boldsymbol{g}$ with

$$
\mathcal{H}_{\eta}(\boldsymbol{g})=\left(\begin{array}{lll}
\frac{\partial I_{\eta}(\boldsymbol{g})}{\partial x \partial x} & \frac{\partial I_{\eta}(\boldsymbol{g})}{\partial x \partial y} & \frac{\partial I_{\eta}(\boldsymbol{g})}{\partial x \partial z} \\
\frac{\partial I_{\eta}(\boldsymbol{g})}{\partial y \partial x} & \frac{\partial I_{\eta}(\boldsymbol{g})}{\partial y \partial y} & \frac{\partial I_{\eta}(\boldsymbol{g})}{\partial x \partial z} \\
\frac{\partial I_{\eta}(\boldsymbol{g})}{\partial z \partial x} & \frac{\partial I_{\eta}(\boldsymbol{g})}{\partial z \partial y} & \frac{\partial I_{\eta}(\boldsymbol{g})}{\partial z \partial z}
\end{array}\right)
$$

where $I_{\eta}(\boldsymbol{g})$ is the 3D image data convolved with a Gaussian with width $\eta$ at point $\boldsymbol{g}$. 

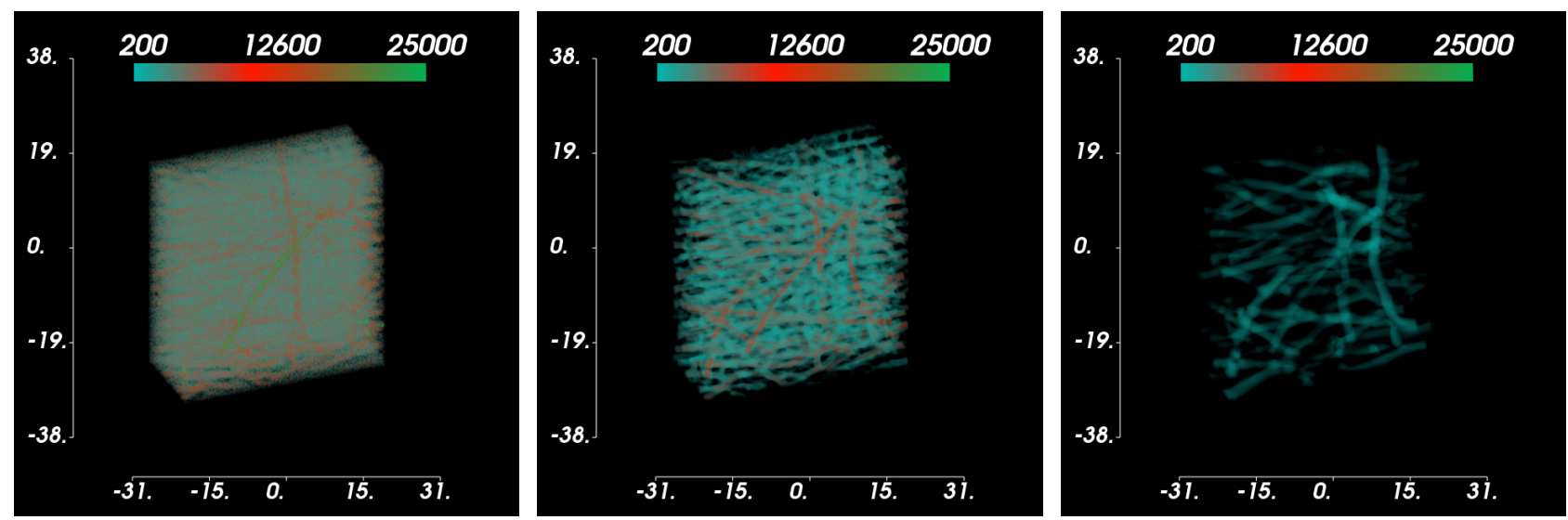

Figure 3: Volume rendering after the fibre enhancement process for exemplar scales $(\eta \in$ $(0.15,0.5,1.5))$ for the aligned fibre sample with scale increasing from left to right.
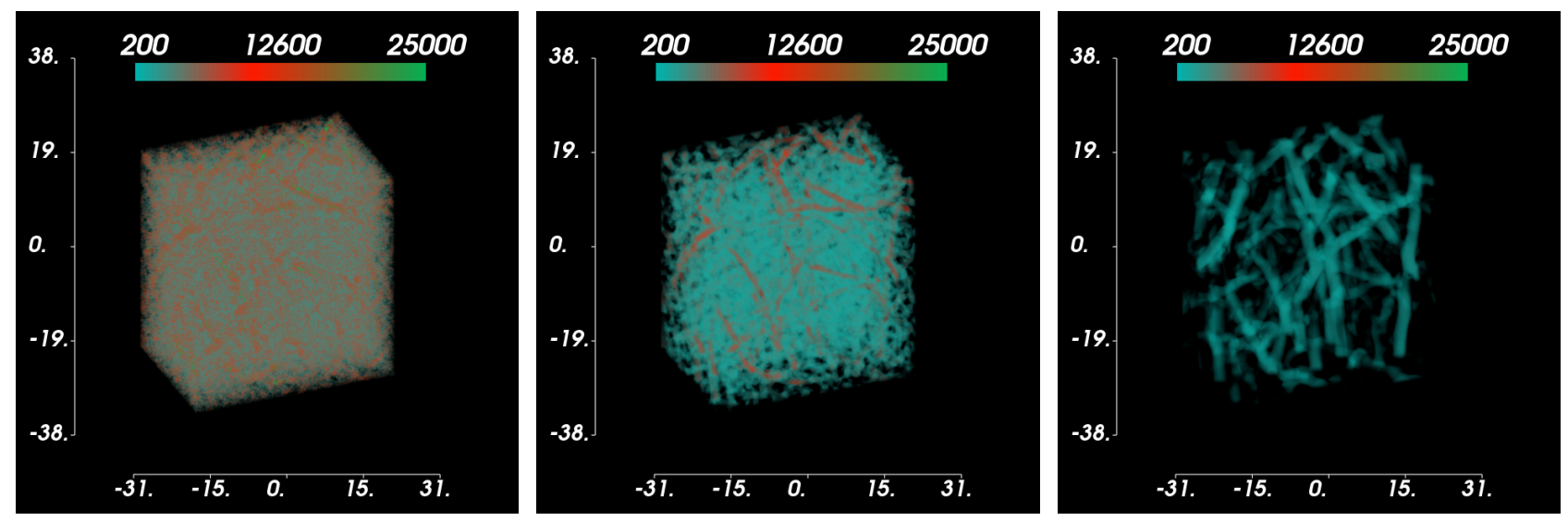

Figure 4: Volume rendering after the fibre enhancement process for exemplar scales $(\eta \in$ $(0.15,0.5,1.5))$ for the randomly distributed fibre sample with scale increasing from left to right.

The Gaussian smoothing kernel can be considered to enable structures, at a particular scale, to be enhanced and also to smooth away structures smaller than the given scale. The second derivatives of the smoothed image data, convolved with a Gaussian kernel can be approximated with the convolution of the image data with the analytically derived second derivative of a Gaussian due to the linear space invariant property of this combined process.

To save space, the scale notation $\eta$ is not included any further. The eigenvalues $\lambda_{1, g}, \lambda_{2, g}$ and $\lambda_{3, \boldsymbol{g}}$ of $\mathcal{H}(\boldsymbol{g})$ are then determined, i.e. $\mathcal{H}(\boldsymbol{g})=U_{\boldsymbol{g}} \Lambda_{\boldsymbol{g}} U_{\boldsymbol{g}}^{\mathrm{T}}$ where $U_{\boldsymbol{g}}$ is an eigenvector matrix and $\Lambda_{\boldsymbol{g}}$ is the diagonal matrix of eigenvalues. Bright tubular structures can be detected by finding points where $\lambda_{3, \boldsymbol{g}} \approx 0, \lambda_{2, \boldsymbol{g}} \ll 0$ and $\lambda_{1, \boldsymbol{g}} \ll 0$ (see e.g. (Sato et al., 1998)). Tubular structures can therefore be enhanced with (from (Sato et al., 1998))

$$
V(\boldsymbol{g})=\left\{\begin{array}{cc}
0 & \text { if } \kappa<0 \\
\kappa \exp \left(-\frac{\lambda_{3, \boldsymbol{g}}^{2}}{2 \alpha \kappa}\right) & \text { elsewhere; }
\end{array}\right.
$$

where $\kappa=\min \left(-\lambda_{1, \boldsymbol{g}},-\lambda_{2, \boldsymbol{g}}\right)$. Exemplar results of applying (2) can be seen in Figs. 3 and 4.

A binary map is created $B(\boldsymbol{g})$ taking a value of 1 for points that are good candidates for a fibre if $V(\boldsymbol{g})$ exceeds a threshold $\tau_{\eta}$, i.e.

$$
B(\boldsymbol{g})= \begin{cases}1 & \text { if } \quad V(\boldsymbol{g}) \geq \tau_{\eta} \\ 0 & \text { elsewhere }\end{cases}
$$




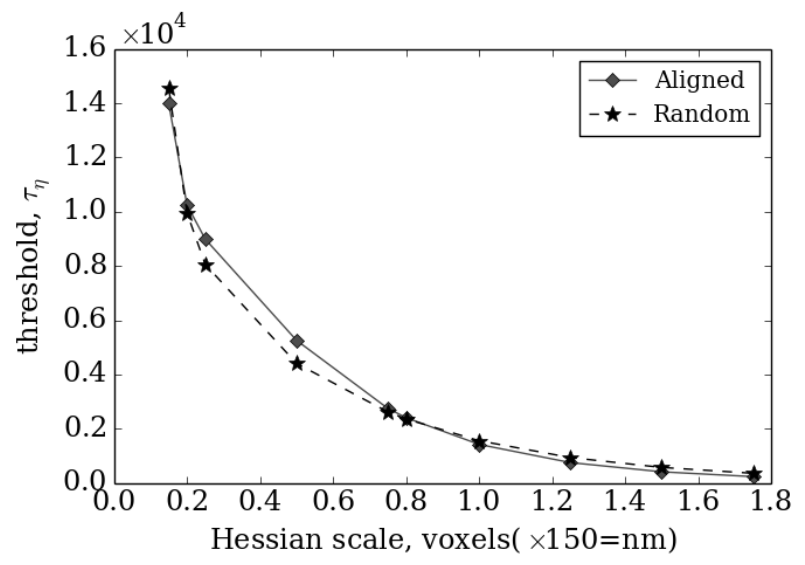

Figure 5: Illustration for a range of different scales for samples with randomly distributed fibres and aligned fibres showing how the threshold $\tau_{\eta}$ varies as a function of scale. These resulting threshold values were used to create the binary maps as in (3).

The threshold $\tau_{\eta}$ is dependent on the scale because the statistical properties of $V(\boldsymbol{g})$ vary dependent on the scale. The threshold is therefore made a function of the percentile of the statistical properties of $V(\boldsymbol{g})$, i.e.

$$
\tau_{\eta}=F_{V}^{-1}(p=0.97)
$$

where $F^{-1}$ is the inverse of the cumulative distribution function of $V(\boldsymbol{g})$. An illustration of the values of $\tau_{\eta}$ for a range of scales can be seen in Fig. 5 for both a randomly distributed sample of fibres and a sample with aligned fibres. This plot illustrates that, for these two fibre samples, the determined threshold follows a very similar curve, illustrating similar statistical properties between sample types.

Connected points in the resulting binary volume $B(\boldsymbol{g})$ consisting of more than a few voxels can be considered to be possible fibre candidates. Each set of connected points possess tubular like image properties at a particular location for a particular scale $\eta$. For each set of connected points, referred to here as the $k^{\text {th }}$ cluster is given by $C_{k}=\left\{\boldsymbol{g}_{i} \mid(\exists j)\left[B\left(\boldsymbol{g}_{i}\right) \wedge B\left(\boldsymbol{g}_{j}\right) \wedge\left\langle\boldsymbol{g}_{i}, \boldsymbol{g}_{j}\right\rangle \leq d\right]\right\}$ where $d$ is a threshold distance. This distance is typically given by the voxel size on a single dimension scaled by $\sqrt{3}$ for 26 connectivity. Once individual sets of connected points have been identified, further processing is necessary to determine whether these candidate fibre locations represent locations at which a measurement, such as fibre diameter or fibre orientation can be accurately recorded. Therefore the major and minor axes of each cluster are computed which is described next. The major and minor axes are then used to perform further processing to compare the correlative symmetry in the minor axes.

\section{Major and Minor Axes Determination}

The major axis of a fibre can be used to characterise the orientation of the fibre and the minor axes can be used to determine the directions over which the diameter of a fibre should be calculated. Therefore, it can be seen that the major and minor axes are important for characterising fibres at discrete locations in the image data. The major and minor axes for an individual cluster can be determine from the eigen vectors of the spatial sample covariation matrix of the points that characterise a cluster. Therefore the sample covariance matrix for each cluster is computed

$$
\mathbf{Q}_{k}=\frac{1}{\left|C_{k}\right|-1} \sum_{\forall \boldsymbol{g}_{i} \in C_{k}}\left(\boldsymbol{g}_{i}-\boldsymbol{\mu}_{k}\right)\left(\boldsymbol{g}_{i}-\boldsymbol{\mu}_{k}\right)^{\mathrm{T}}
$$

where $\boldsymbol{\mu}_{k}$ is the centre of cluster $k$, i.e. the mean computed with $\boldsymbol{\mu}_{k}=\frac{1}{\left|C_{k}\right|} \sum_{\forall \boldsymbol{g}_{i} \in C_{k}} \boldsymbol{g}_{i}$. 
Eigenvector decomposition of the covariance matrices for each cluster is then performed $\mathrm{Q}_{k}=$ $R_{k} \Sigma_{k} R_{k}^{\mathrm{T}}$ where $R_{k}$ and $\Sigma_{k}$ are a matrix of eigenvectors and a matrix of eigenvalues respectively for the $k^{\text {th }}$ cluster of connected voxels. The eigenvectors indicate here candidate major and minor axes on which the eigenvalues describe the amount of spread. The major axis is the direction given by the eigenvector with the greatest eigenvalue, i.e. $\lambda_{1, k}$ where $\lambda_{1, k} \geq \lambda_{2, k} \geq \lambda_{3, k}$. A fibre would be expected to have the longest length along this axis; whilst the two eigenvectors corresponding to the two smaller eigenvalues $\lambda_{2, k}$ and $\lambda_{3, k}$ would be expected to form the minor axes of the fibre because they are orthogonal to the major axis. Due to the statistical nature of the aforementioned processes, it is likely that a number of candidate fibres may need to be rejected. This might be because of a number of reasons, such as clusters of high intensity points that may be somewhat fibre like but in actual fact are poor candidates for this sampling process. Another reason might be due to a fibre being highly irregular but still tubular and with high intensity. The above fibre enhancement stage may still identify those as candidate fibres but are likely to act as poor candidates for subsequent statistical analysis of fibre properties.

\section{Fibre Candidacy}

A further stage is therefore needed to determine if a fibre conforms to a number of requirements that would enable useful properties to be associated with an individual fibre. These requirements are: the two minor axes' estimated diameters of the fibre should be approximately equal; and the two minor axes' intensity profiles $I_{l, 2, k}$ and $I_{l, 3, k}$ should be similar where the line profiles have $L$ voxels and individual voxels, indexed by $l \in L$ are determined using Bresenham's line algorithm (Bresenham, 1965). The similarity of the line profiles can include similarity of the maximum intensities along each profile $I_{2, k}^{\max }, I_{3, k}^{\max }$ and similarity of the minimum intensities along each profile $I_{2, k}^{\min }, I_{3, k}^{\min }$. The maximums and minimums of the line profiles for the minor axes can be determined with

$$
\begin{aligned}
I_{2, k}^{\max } & =\max _{l \in[0, L-1]} I_{l, 2, k}, \quad I_{3, k}^{\max }=\max _{l \in[0, L-1]} I_{l, 3, k} ; \text { and similarly } \\
I_{2, k}^{\min } & =\min _{l \in[0, L-1]} I_{l, 2, k}, \quad I_{3, k}^{\min }=\min _{l \in[0, L-1]} I_{l, 3, k} \text { respectively. }
\end{aligned}
$$

The similarity of the maximum $I_{2, k}^{\max }, I_{3, k}^{\max }$ and minimum values $I_{2, k}^{\min }, I_{3, k}^{\min }$ for the two minor axes $(2,3)$ for cluster $k$ can then be calculated with

$$
\begin{gathered}
D_{k, \text { max }}=\frac{\left|2\left(I_{2, k}^{\max }-I_{3, k}^{\max }\right)\right|}{\left|\left(I_{2, k}^{\max }+I_{3, k}^{\max }\right)\right|} \text { and } \\
D_{k, \text { min }}=\frac{\left|2\left(I_{2, k}^{\min }-I_{3, k}^{\min }\right)\right|}{\left|\left(I_{2, k}^{\min }+I_{3, k}^{\min }\right)\right|} .
\end{gathered}
$$

If the maximum similarity has a value below e.g. $D_{k, \max } \leq 0.02$ then we consider it to be approximately equal as the maximum should coincide with the centre of the fibre through which both axes traverse. However, the minimum value is likely to vary more widely so we found, by empirical experimentation that a value of $D_{k, \text { min }} \leq 0.2$ gave a strong indication of a fibre with some geometric properties that could prove useful in the statistical characterisation of the data.

A further test to determine a candidate fibre's minor axis properties is to compare the intensity profiles for the two minor axes. Correlation in the form of the sample correlation coefficient is used here to compare the line intensity profiles $I_{l, 2, k}$ and $I_{l, 3, k}$ where

$$
\rho_{k_{2,3}}=\frac{\sum_{l=0}^{L-1}\left(I_{l, 2, k}-\mu_{2, k}\right)\left(I_{l, 3, k}-\mu_{3, k}\right)}{(L-1) \eta_{2, k} \eta_{3, k}}
$$

A high correlation e.g. $\rho_{k_{2,3}} \geq 0.9$ would indicate that the intensity line profiles correlate reasonably well which increases the possibility that the candidate fibre is correct. Some comparative line profiles can be seen in Fig. 6. 

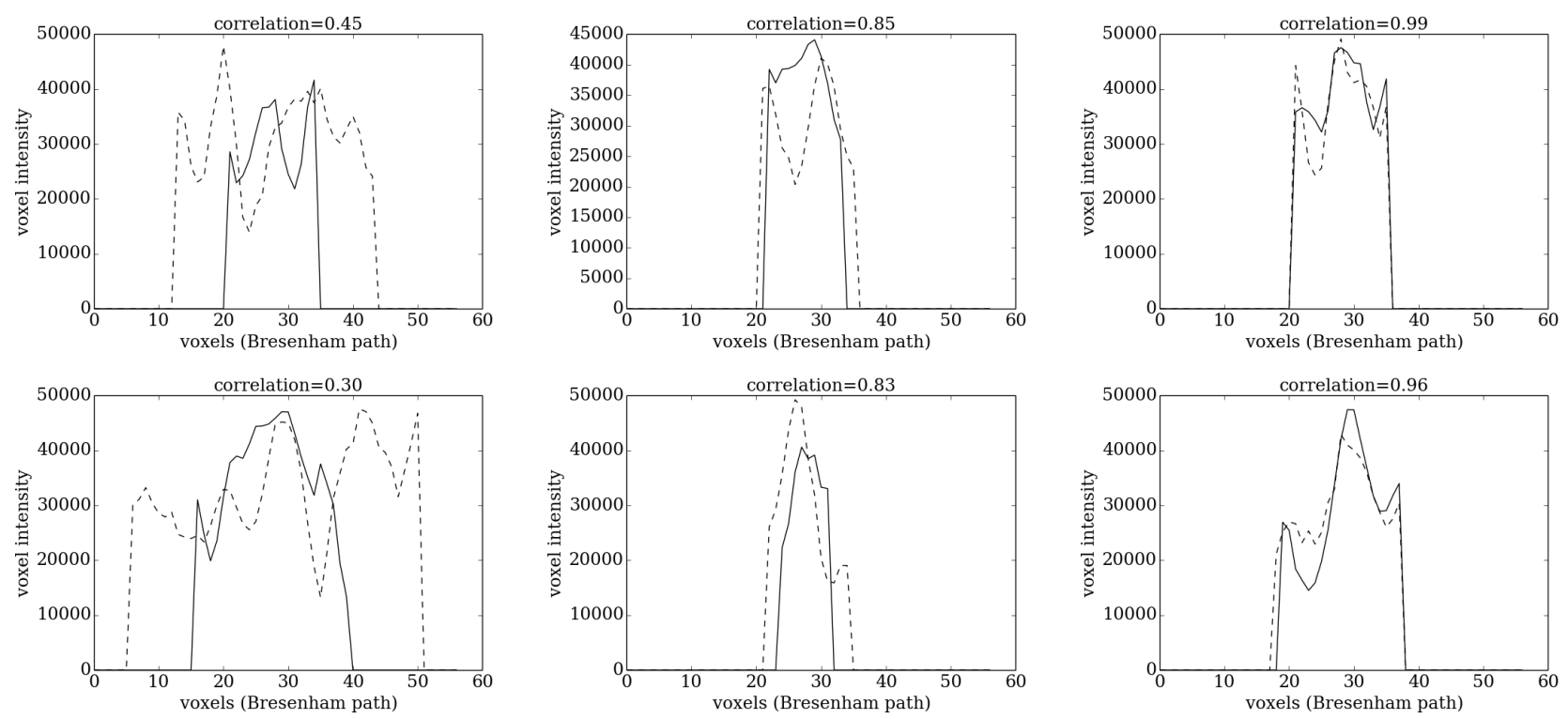

Figure 6: Example plots of some minor axes (orthogonal) line profiles, illustrating line profiles with low correlation and line profiles with high correlation. The line profiles are taken along Bresenham paths Bresenham (1965), stepping through discrete voxel locations. The actual length is computed subsequently. Also note the variation in minima surrounding the central peak (maximum) values in each line profile.

\section{Fibre Diameter Determination}

It might be expected that the diameter of a fibre could potentially be found by finding the point at which the intensity of the fibre drops down to a level comparable to the surrounding medium. However, it is interesting to observe the variation in the minimum intensity levels surrounding each individual fibre. This can be seen illustrated in Fig. 2 and also in the exemplar profiles shown in Fig. 6. The diameter is therefore determined dynamically based on finding the distance from the minimum intensity profile value for both line profiles, which can be considered dual estimates of the radius of each fibre but in orthogonal directions. A further consideration here is regarding the effect of the blurring action of the Point Spread Function (PSF) which will result in an over estimation of the width of a fibre if the limits are taken from the lowest observable points.

Therefore in common with e.g. (Mets et al., 2012), the diameter is taken at the mid-point between the points at which the maximum and minimum are observed. The diameter is thus calculated with

$$
\gamma_{i, \eta}=2 \times\left\langle\boldsymbol{g}_{\text {start }}, \boldsymbol{g}_{\text {end }}\right\rangle \times \frac{\left(n_{\max }-n_{\min }\right)}{L}
$$

where $\boldsymbol{g}_{\text {start }}, \boldsymbol{g}_{\text {end }}$ are the start and end points along the Bresenham path but in voxel coordinates with dimensions (e.g. $\mu \mathrm{m}$ ) respectively; and $n_{\max }-n_{\min }$ and $L$ are the number of voxels along the Bresenham path between the voxels with the maximum and minimum intensities and the total number of voxels along the Bresenham path. The diameter is estimated along both minor axes for every cluster $i$.

The mean and variance of the fibre candidates can be determined for a given Hessian scale with

$$
\begin{gathered}
\mu_{\eta}=\frac{1}{\beta_{\eta}} \sum_{\forall i} \gamma_{i, \eta} ; \\
\sigma_{\eta}^{2}=\frac{1}{\beta_{\eta}} \sum_{\forall i}\left(\gamma_{i, \eta}-\mu_{\eta}\right)^{2}
\end{gathered}
$$

respectively where $\beta_{\eta}$ is the number of selected candidate fibres at scale $\eta$. 
Each diameter estimate $\gamma_{i, \eta}$ will potentially have an error associated with it. Furthermore, the number of detected fibres at any given scale may vary. Any further averaging could potentially benefit by taking into account these factors. Therefore, each diameter estimate $\gamma_{i, \eta}$ is considered to be an instance of a Gaussian distributed random variable, i.e. $\Gamma_{\eta} \in \mathcal{N}\left(\mu_{\eta}, \sigma_{\eta}^{2}\right)$ at scale $\eta$ (see (1)). The probability density is given by

$$
p\left(\gamma_{\eta} \mid \mu_{\eta}, \sigma_{\eta}\right)=\frac{1}{\sqrt{2 \pi \sigma_{\eta}}} \exp \left(-\frac{\left(\gamma_{\eta}-\mu_{\eta}\right)^{2}}{2 \sigma_{\eta}^{2}}\right) .
$$

This helps to model the variation that can be observed for the fibre diameters at each scale $\eta$. A further parameter of interest is the number of detections $\beta_{\eta}$ which can potentially provide an indication of the frequency of fibres at that particular scale with distribution given by (14). This information will result in a range of densities, one for each scale representing the possible ranges of diameters for each scale.

It can often be useful to provide a single estimate of diameter, which can be obtained by combining a weighted combination of the above densities. The weighting would need to take into account the number of detections at each scale, so that, scales with a relatively large number of detections would need to contribute a greater weight to the overall estimate in comparison to scales with relatively few detections. The overall diameter estimate would also need to take into account the spread of the diameter estimates at each scale.

Therefore, an overall diameter estimate, independent of scale can be found with

$$
\hat{\gamma}=\frac{1}{\sum_{\forall \eta} \beta_{\eta} p\left(\gamma_{\eta}=\mu_{\eta}\right)} \sum_{\forall \eta} \beta_{\eta} p\left(\gamma_{\eta}=\mu_{\eta}\right) \mu_{\eta} .
$$

Noting that $p\left(\gamma_{\eta}=\mu_{\eta}\right)=\frac{1}{\sqrt{2 \pi \sigma_{\eta}^{2}}}$ and substituting into (15) along with (14) to get

$$
\hat{\gamma}=\frac{1}{\sum_{\forall \eta} \frac{\beta_{\eta}}{\sqrt{2 \pi \sigma_{\eta}^{2}}}} \sum_{\forall \eta} \frac{\beta_{\eta}}{\sqrt{2 \pi \sigma_{\eta}^{2}}}\left(\frac{1}{\beta_{\eta}} \sum_{\forall i} \gamma_{i, \eta}\right) .
$$

Cancelling terms results in

$$
\hat{\gamma}=\frac{1}{\sum_{\forall \eta} \frac{\beta_{\eta}}{\sigma_{\eta}}} \sum_{\forall \eta}\left(\frac{1}{\sigma_{\eta}} \sum_{\forall i} \gamma_{i, \eta}\right) .
$$

This now provides a way to calculate an overall diameter estimate across scale, taking into account the number of detections and the inherent variability observed at each resolution.

\section{Orientation Distribution and Summarisation}

The orientation distribution is another important characteristic in many fields of science and engineering when considering the distribution of fibres in a material. It can be characterised purely as a $2 \mathrm{D}$ distribution in terms of the spherical coordinate system consisting the in-plane $\theta \in[0 \pi]$ (or polar) angle and out-of-plane angle $\phi \in\left[-\frac{\pi}{2} \frac{+\pi}{2}\right]$. A spherical coordinate system would also require a radius $r$ but this is not usually incorporated into the orientation distribution. For a given vector, the orientation parameters $(r, \theta, \phi)$ can be computed with $r=\sqrt{x^{2}+y^{2}+z^{2}} ; \theta=\tan ^{-1}\left(\frac{y}{x}\right)$; and $\phi=\cos ^{-1}\left(\frac{z}{r}\right)$. The angle pair $(\theta, \phi)$ can be approximated via discretisation resulting in $(n, m)$ in combination with a distribution $P_{N, M}(n, m)$ where $N, M$ are the resolution over which the discretization $n, m$ is taken. The orientation distribution can then be summarised over $P_{N, M}(n, m)$ with a multiscale Shannon's entropy measure, as shown in (Chiverton et al., 2017)

$$
H_{\mathrm{ms}}=\frac{1}{|\mathfrak{N}||\mathfrak{M}|} \sum_{N \in \mathfrak{N}} \sum_{M \in \mathfrak{M}} \sum_{n \in N} \sum_{m \in M} P_{N, M}(n, m) h_{N, M}(n, m)
$$




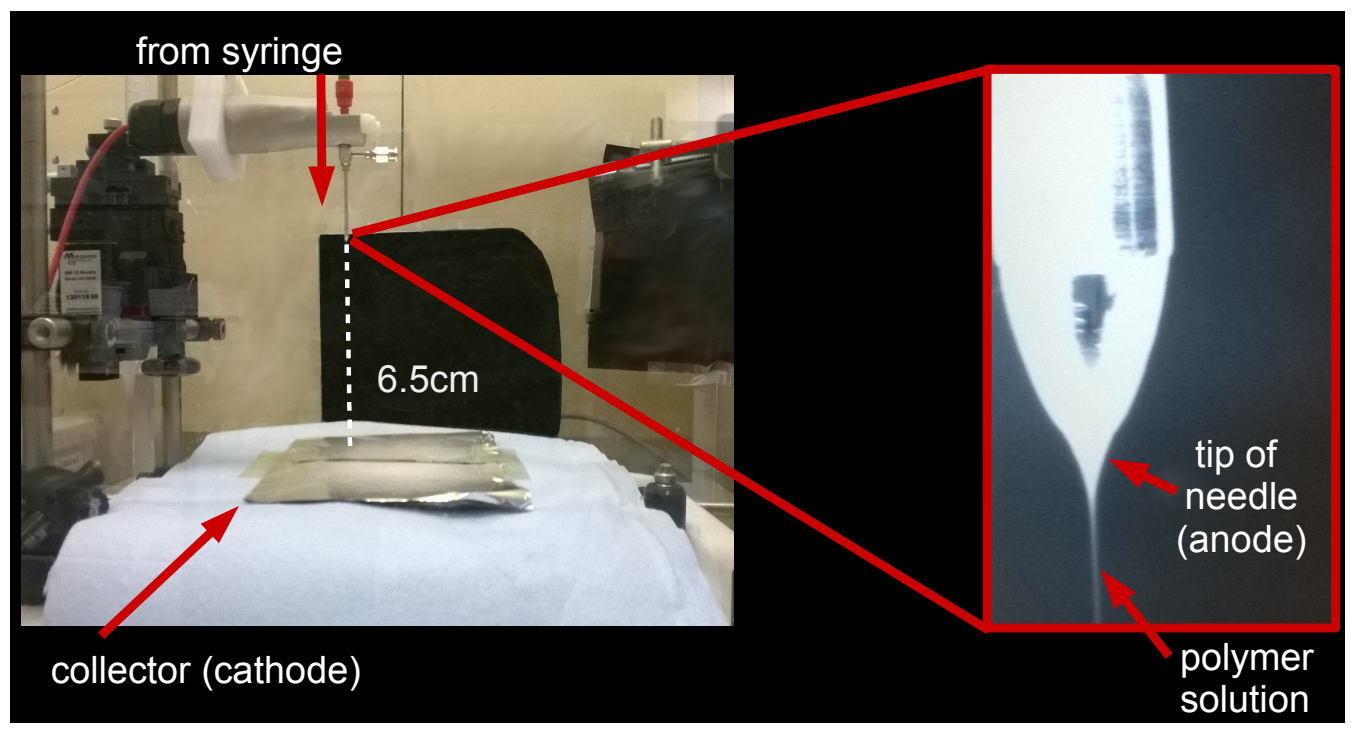

Figure 7: Overview of the experimental setup used to perform electrospinning (left) and close up of the needle tip with the polymer solution being pumped so that it exits the needle tip (right).

where the self-information is given by $h_{N, M}(n, m)=-\log _{2}\left(P_{N, M}(n, m)\right) ; N$ and $M$ are the scales over which $(n, m)$ are discretised; and $\mathfrak{N}$ and $\mathfrak{M}$ are the sets of scales over which the multiscale entropy is calculated.

\section{Experimental Details}

Electrospun poly(caprolactone) (PCL) nano-fibre samples were prepared. PCL (80 KDa, 1.5g) was dissolved in chloroform $(7.5 \mathrm{ml})$ under vigorous stirring, once completely dissolved, methanol (2.5 $\mathrm{ml}$ ) was added and thoroughly mixed. Solutions were electrospun using the Spraybase device by Profector Life Science Ltd. (Ireland) connected to a custom made capillary nozzle by Rame-hart Instruments Co. (NJ, US) with an internal diameter of $508 \mu \mathrm{m}$ (Fig. 7). The capillary nozzle was connected to a high-voltage supply ( $\max 30 \mathrm{kV}$ ) operated at $6.5 \mathrm{kV}$ and positioned at $11 \mathrm{~cm}$ distance from the collector. Solutions were perfused through the nozzle via a syringe pump (World Precision Instruments, $\mathrm{FL}, \mathrm{US}$ ) ran at $6 \mathrm{ml} / \mathrm{h}$ and fitted with a $5 \mathrm{ml}$ gas-tight glass syringe (Hamilton). A total volume of $2 \mathrm{ml}$ was injected. All experiments were carried out at ambient temperature. Fibres were collected on a grounded aluminium foil. To achieve a random distribution of the fibres, a continuous collector was used. For the aligned fibres, a collector with a $0.5 \mathrm{~cm}$ wide slit was used. An overview of the setup of the experiment can be seen in Fig. 7.

The PCL electrospun fibre samples were then scanned with a Zeiss Xradia Versa 510 Microtomography X-Ray Computer Tomography (XCT) system with an isotropic voxel resolution of $0.15^{3} \mu \mathrm{m}$. The resulting scan consisted of $1804 \times 1956 \times 300$ voxels or, equivalently a $270.6 \times 293.4 \times 45 \mu \mathrm{m}^{3}$ volume. A sub-volume or region-of-interest (see Fig. 1) was then created by cropping the initial scanned volume consisting of $200 \times 300 \times 285$ voxels to simplify subsequent analysis.

SEM images were also obtained for gold-coated samples (sputter coater Q150R, Quorom Technologies) with a Zeiss EVO MA10 scanning electron microscope with electron back-scatter capability. Exemplar SEM images can be seen in Fig. 8.

Software was written in C++ using functionality from ITK and VTK, see (Johnson et al., 2013; Schroeder et al., 2006). Scripting including data plotting was performed with Python and Matplotlib (Hunter, 2007). An outline of the overall system approach can be seen in Fig. 9. 

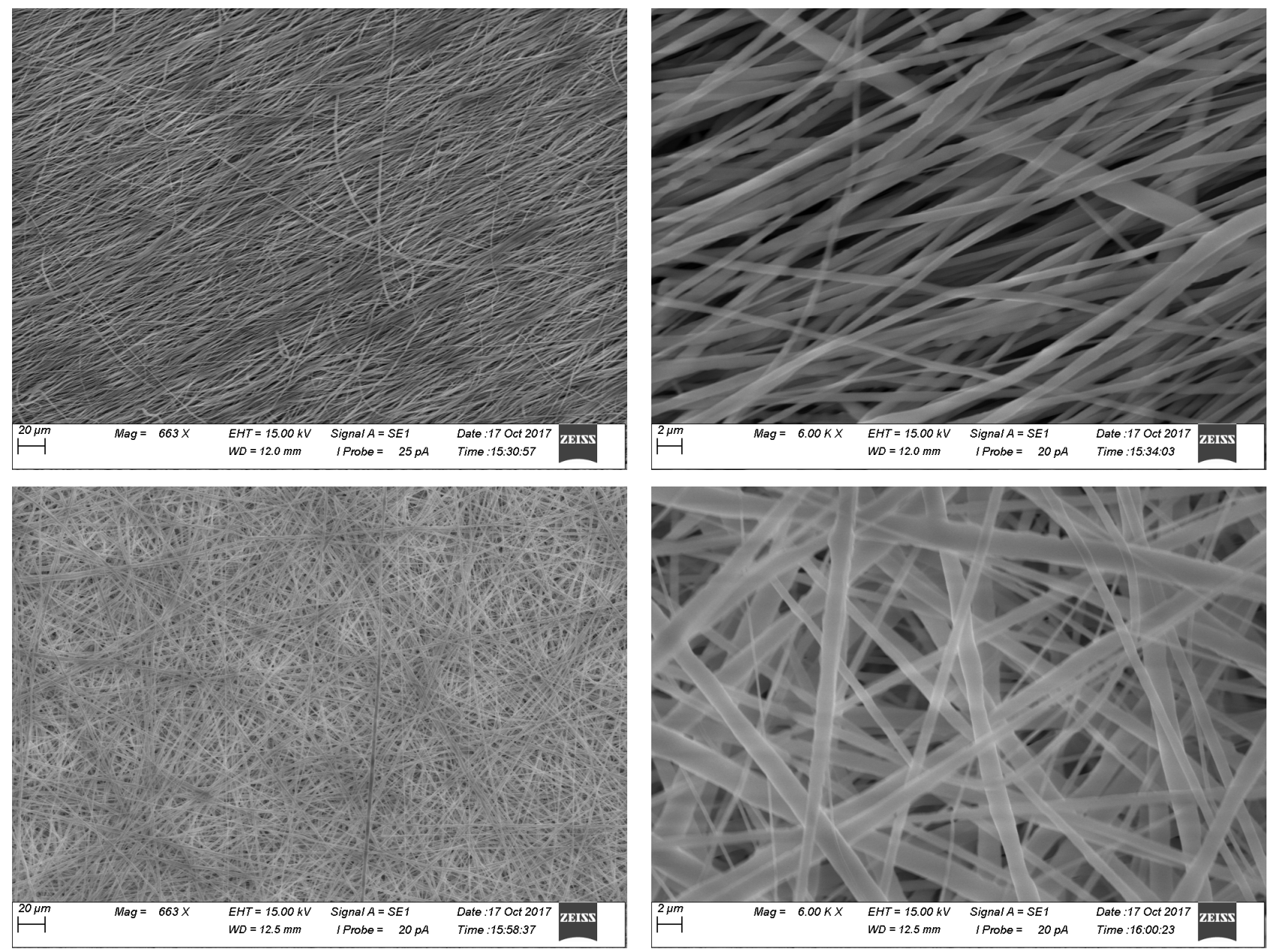

Figure 8: SEM images of an aligned fibre sample (top row) and a randomly distributed fibre sample (bottom row) at two different resolutions for the two samples.

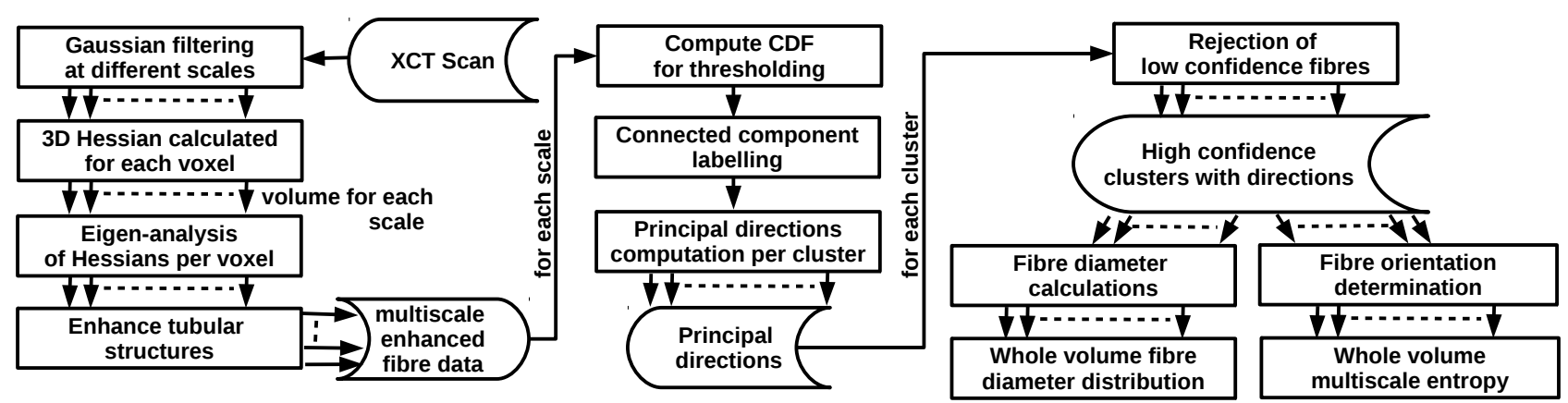

Figure 9: An outline of the overall system approach as described here in this work. 

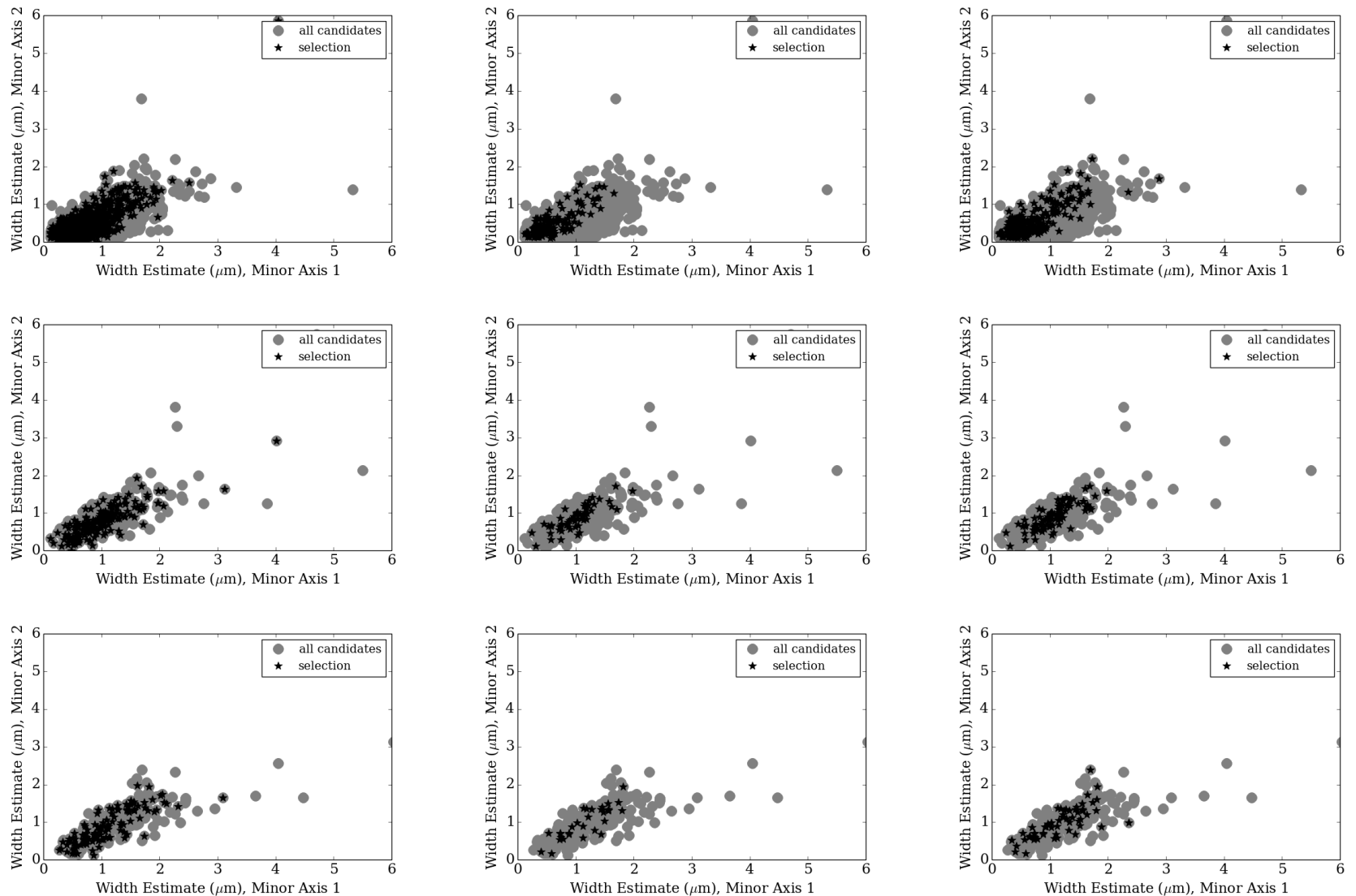

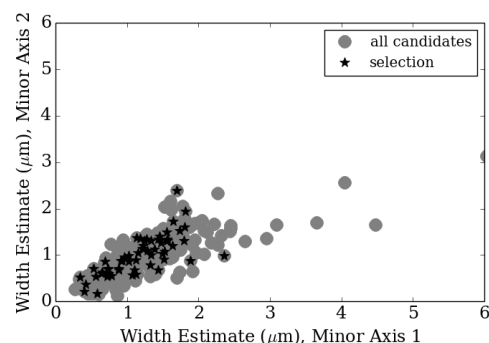

Figure 10: Comparing, for a sample with aligned fibres, via scatter plots, consistency of estimates of the diameter $\gamma$ in (11), across a number of scales $(\eta \in(0.15,0.50,0.75))$ for the different selection criteria. Left column: selection using minimums and maximums of line profiles, i.e. (8) \& (9); middle column: selection using the minimums and maximums of line profiles (8), (9) and the correlation between line profiles i.e. (10); right column: selection using just correlation, (10). The scatter plots using correlation appear to be the most effective at reducing the number of outliers here.

\section{Results}

Comparisons were made in the effectiveness of rejecting outliers in the fibre diameter estimation process for the two orthogonal axes, as shown in Figs. 10 and 11.

A table of mean estimated fibre diameters for the different fibre selection techniques is shown in Table 1 . Here it can be seen a range of different diameters have been estimated as a result of the different selection processes.

\section{Scale Selection}

It is interesting to note that the results shown in Table 1 are for a single scale of the Hessian matrix in (1). It would be expected that the diameter estimates might vary at different scales. Therefore the same process of estimating the diameter of the fibres was repeated but using a number of different scales in (1) as illustrated in Figs. 3 and 4. The resulting diameter estimates can be seen in Figs. 12(a) and 12(b). It can be seen that at greater scales the standard deviation of the estimates generally increases. Furthermore, as might be expected, at greater scales, the system has detected fibres with greater diameters however, because of the large error on these estimates, it could equally mean that the true fibre diameters should actually be much less than the ones estimated using the mean. 

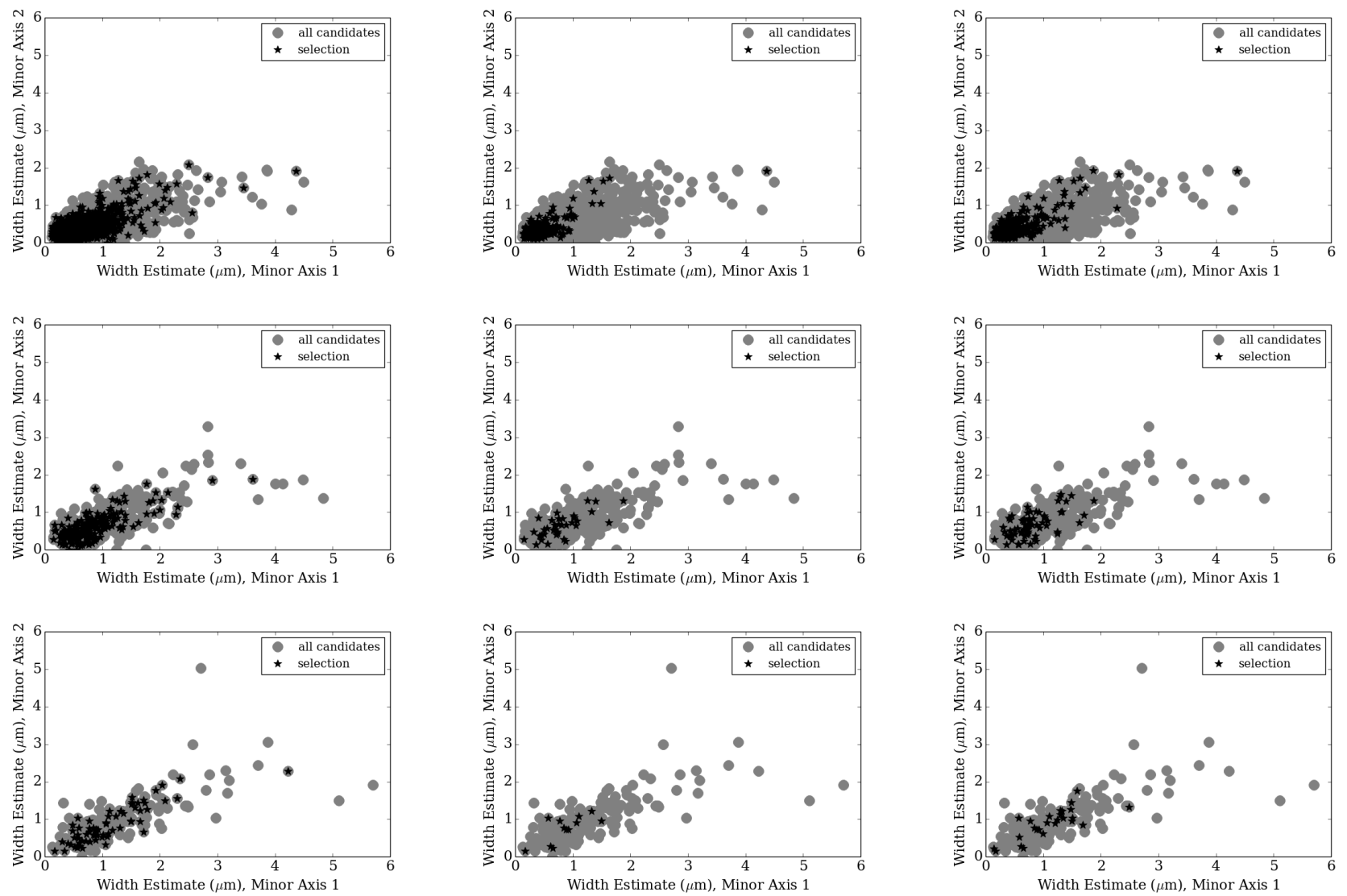

Figure 11: Comparing, for a sample with randomly distributed fibres, via scatter plots, consistency of estimates of the diameter $\gamma$ in (11), across a number of scales $(\eta \in(0.15,0.50,0.75))$ for the different selection criteria. Left column: selection using minimums and maximums of line profiles, i.e. (8) \& (9); middle column: selection using the minimums and maximums of line profiles (8), (9) and the correlation between line profiles i.e. (10); right column: selection using just correlation, (10). The scatter plots using correlation appear to be the most effective at reducing the number of outliers here.

\begin{tabular}{|c|c|c|c|c|c|}
\hline selection & axis & $\begin{array}{c}\text { Align } \\
\text { mean } \\
\mu \mathrm{m}\end{array}$ & $\begin{array}{c}\text { d Fibres } \\
\text { st. dev. } \\
\mu \mathrm{m}\end{array}$ & $\begin{array}{l}\text { Randd } \\
\text { mean } \\
\mu \mathrm{m}\end{array}$ & $\begin{array}{c}\text { n Fibres } \\
\text { st. dev. } \\
\mu \mathrm{m}\end{array}$ \\
\hline none & $\begin{array}{c}\text { minor axis } 1 \\
\text { minor axis } 2 \\
\text { axis mean }\end{array}$ & $\begin{array}{l}0.69 \\
0.43 \\
0.56\end{array}$ & $\begin{array}{l}0.57 \\
0.35 \\
0.42 \\
\end{array}$ & $\begin{array}{l}0.63 \\
0.38 \\
0.51 \\
\end{array}$ & $\begin{array}{l}0.40 \\
0.25 \\
0.29 \\
\end{array}$ \\
\hline $\begin{array}{c}\text { max. \& min. } \\
(8),(9)\end{array}$ & $\begin{array}{c}\text { minor axis } 1 \\
\text { minor axis } 2 \\
\text { axis mean }\end{array}$ & $\begin{array}{l}0.59 \\
0.38 \\
0.49\end{array}$ & $\begin{array}{l}0.36 \\
0.29 \\
0.30\end{array}$ & $\begin{array}{l}0.54 \\
0.34 \\
0.44\end{array}$ & $\begin{array}{l}0.28 \\
0.19 \\
0.22\end{array}$ \\
\hline $\begin{array}{l}\text { max. \& min. } \\
\& \text { corr. } \\
(8),(9),(10)\end{array}$ & $\begin{array}{c}\text { minor axis } 1 \\
\text { minor axis } 2 \\
\text { axis mean }\end{array}$ & $\begin{array}{l}0.54 \\
0.44 \\
0.49\end{array}$ & $\begin{array}{l}0.29 \\
0.29 \\
0.28\end{array}$ & $\begin{array}{l}0.47 \\
0.38 \\
0.43\end{array}$ & $\begin{array}{l}0.32 \\
0.25 \\
0.27\end{array}$ \\
\hline $\begin{array}{l}\text { corr. } \\
(10)\end{array}$ & $\begin{array}{c}\text { minor axis } 1 \\
\text { minor axis } 2 \\
\text { axis mean }\end{array}$ & $\begin{array}{l}0.62 \\
0.49 \\
0.56\end{array}$ & $\begin{array}{l}0.38 \\
0.35 \\
0.35\end{array}$ & $\begin{array}{l}0.51 \\
0.41 \\
0.46\end{array}$ & $\begin{array}{l}0.33 \\
0.26 \\
0.28\end{array}$ \\
\hline
\end{tabular}

Table 1: Results for the fibre diameter estimation process involving differing candidate fibre selections for $\eta=0.15$. 

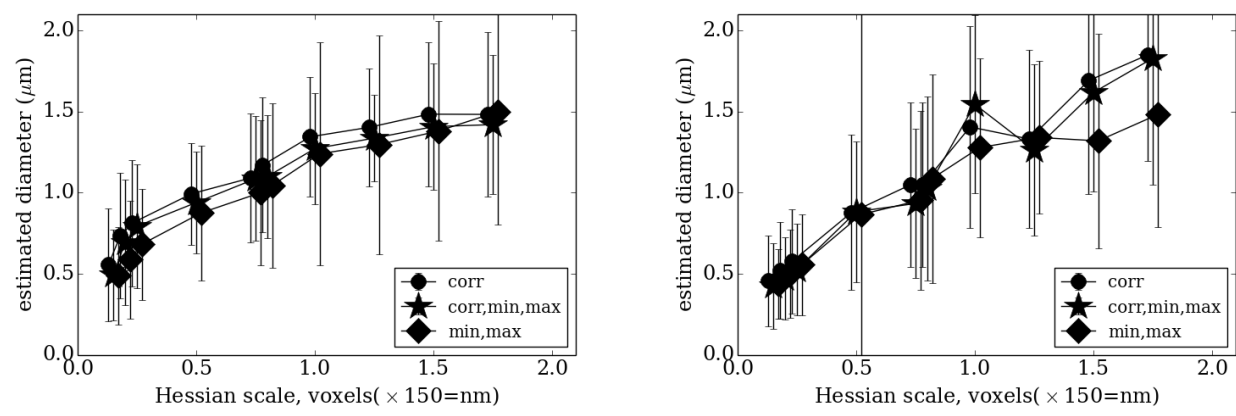

Figure 12: Estimated diameters using mean estimates of $\mu_{\eta}$ from (12) as a function of a range of different scale values $\eta$ as used in the computation of the Hessian matrix in (1). The estimates are for a sample with aligned fibres (left) and one with randomly distributed fibres (right). The error bars indicate $\pm(\times 1)$ standard deviation of the estimated diameters.

\begin{tabular}{|c|c|c|c|c|c|c|}
\hline & \multicolumn{3}{|c|}{ Aligned Fibres } & \multicolumn{3}{c|}{ Random Fibres } \\
scale & mean & st. dev. & count & mean & st. dev. & count \\
$\eta(\mu \mathrm{m})$ & $\mu_{\eta}(\mu \mathrm{m})$ & $\sigma(\mu \mathrm{m})$ & $\beta_{\eta}$ & $\mu_{\eta}(\mu \mathrm{m})$ & $\sigma(\mu \mathrm{m})$ & $\beta_{\eta}$ \\
\hline \hline 0.15 & 0.49 & 0.30 & 1821 & 0.44 & 0.22 & 5030 \\
0.25 & 0.68 & 0.34 & 383 & 0.56 & 0.31 & 1966 \\
0.5 & 0.88 & 0.41 & 187 & 0.87 & 1.27 & 310 \\
0.75 & 1.00 & 0.45 & 114 & 0.95 & 0.55 & 129 \\
1.0 & 1.24 & 0.69 & 67 & 1.28 & 0.55 & 61 \\
1.25 & 1.30 & 0.67 & 43 & 1.34 & 0.47 & 59 \\
1.5 & 1.38 & 0.68 & 30 & 1.32 & 0.66 & 43 \\
1.75 & 1.50 & 0.69 & 17 & 1.48 & 0.69 & 23 \\
\hline
\end{tabular}

Table 2: Scale dependent results using min. and max. selection only for mean width and error estimation.

Means, standard deviations and fibre detection counts for the three fibre selection processes can be seen in Tables 2, 3 and 4 . The estimated means $\mu_{\eta}$ using (12) vary considerably dependent on scale. However, the standard deviations also vary and so do the overall counts of detected fibres. This is a further interesting result, in terms of the number of detected fibres as a function of scale and the selection technique. It can be seen that as the scale increases, far fewer fibres are detected. The larger scales will therefore contribute less to the overall diameter estimates to be seen shortly. This will also be further combined with the effect of the relatively larger standard deviations that can be seen at greater scales. The standard deviation increases for an increasing value of scale. Furthermore the detection count reduces for increasing scale. This means that the mean diameter estimates become less prominent in a technique based on (17), the results of which can be seen illustrated in Fig. 13 where the individual scales, modelled as Gaussians combine to form a final point estimate.

The final combined density estimates, also shown in Fig. 13 each have a clearly defined modal point. The overall estimated means and modes can be seen in Table 5 for the 3 different candidate fibre selection techniques. The mean results appear to suggest a fibre diameter of $\hat{\gamma} \in[0.5,0.55] \mu \mathrm{m}$ for the random fibres whereas the mode estimates appear to suggest modal fibre diameters in the range of $[0.47,0.54] \mu \mathrm{m}$. For the aligned fibres, the ranges are wider, for the mean between $[0.59,0.81]$ and for the mode between $[0.54,0.74]$.

Further observations can be made regarding the diameter estimates at different scales, as seen in Tables 2, 3 and 4, indicating the preferential ability at different scales to detect fibres of different widths. 


\begin{tabular}{|c|c|c|c|c|c|c|}
\hline & \multicolumn{3}{|c|}{ Aligned Fibres } & \multicolumn{3}{c|}{ Random Fibres } \\
scale & mean & st. dev. & count & mean & st. dev. & count \\
$\eta(\mu \mathrm{m})$ & $\mu_{\eta}(\mu \mathrm{m})$ & $\sigma(\mu \mathrm{m})$ & $\beta_{\eta}$ & $\mu_{\eta}(\mu \mathrm{m})$ & $\sigma(\mu \mathrm{m})$ & $\beta_{\eta}$ \\
\hline \hline 0.15 & 0.49 & 0.28 & 201 & 0.43 & 0.27 & 597 \\
0.25 & 0.79 & 0.38 & 75 & 0.53 & 0.28 & 267 \\
0.5 & 0.94 & 0.31 & 57 & 0.88 & 0.43 & 69 \\
0.75 & 1.09 & 0.38 & 31 & 0.93 & 0.46 & 33 \\
1.0 & 1.27 & 0.34 & 20 & 1.54 & 0.55 & 16 \\
1.25 & 1.34 & 0.27 & 10 & 1.26 & 0.53 & 16 \\
1.5 & 1.41 & 0.39 & 5 & 1.62 & 0.61 & 12 \\
1.75 & 1.42 & 0.43 & 6 & 1.83 & 0.78 & 5 \\
\hline
\end{tabular}

Table 3: Scale dependent results using correlation, min. and max. selection for mean width and error estimation.

\begin{tabular}{|c|c|c|c|c|c|c|}
\hline & \multicolumn{3}{|c|}{ Aligned Fibres } & \multicolumn{3}{c|}{ Random Fibres } \\
scale & mean & st. dev. & count & mean & st. dev. & count \\
$\eta(\mu \mathrm{m})$ & $\mu_{\eta}(\mu \mathrm{m})$ & $\sigma(\mu \mathrm{m})$ & $\beta_{\eta}$ & $\mu_{\eta}(\mu \mathrm{m})$ & $\sigma(\mu \mathrm{m})$ & $\beta_{\eta}$ \\
\hline \hline 0.15 & 0.56 & 0.35 & 332 & 0.46 & 0.28 & 929 \\
0.25 & 0.81 & 0.39 & 104 & 0.58 & 0.32 & 448 \\
0.5 & 0.99 & 0.31 & 89 & 0.88 & 0.48 & 129 \\
0.75 & 1.09 & 0.40 & 58 & 1.05 & 0.51 & 57 \\
1.0 & 1.34 & 0.37 & 36 & 1.41 & 0.62 & 33 \\
1.25 & 1.40 & 0.36 & 24 & 1.33 & 0.55 & 34 \\
1.5 & 1.48 & 0.44 & 15 & 1.69 & 0.70 & 25 \\
1.75 & 1.48 & 0.51 & 18 & 1.85 & 0.65 & 16 \\
\hline
\end{tabular}

Table 4: Scale dependent results using correlation only for mean width and error estimation.

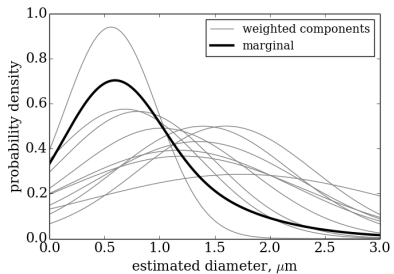

(c) No selection.

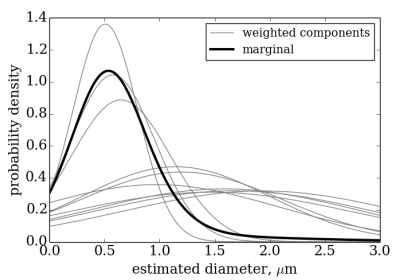

(g) No selection.

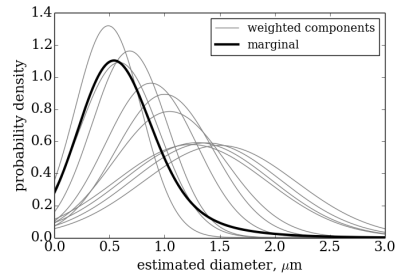

(d) Selection using (9).

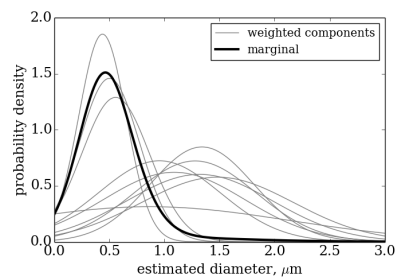

(h) Selection using (9).
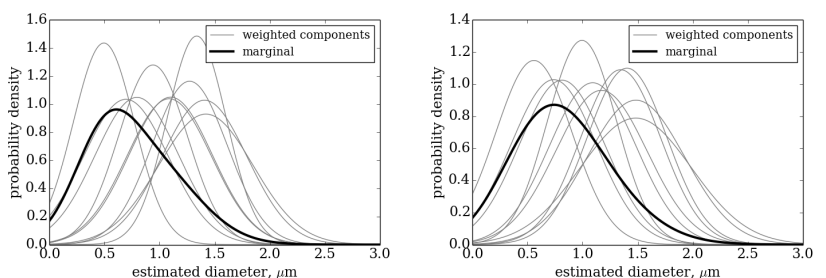

(e) Selection using (8), (9) $\&(10)$.

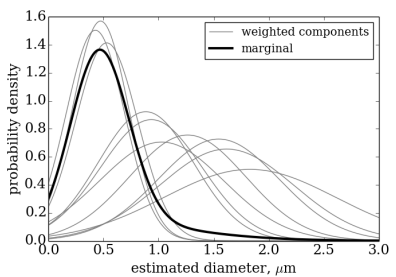

(f) Selection using just $(10)$.

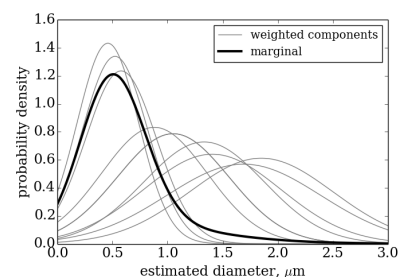

(i) Selection using (8), (9) (j) Selection using just (10). $\&(10)$.

Figure 13: Illustration of the effects of the estimated diameters using (17) for samples with aligned fibres (top row) and randomly distributed fibres (bottom row), where the estimated parameters at each scale contribute a single Gaussian kernel. The final combined density estimates are also shown. 


\begin{tabular}{|c|c|c|c|c|}
\hline \multirow{2}{*}{ selection } & \multicolumn{2}{|c|}{ Aligned } & \multicolumn{2}{c|}{ Random } \\
& $\begin{array}{c}\text { mean } \\
\hat{\gamma}(\mu \mathrm{m})\end{array}$ & $\begin{array}{c}\text { mode } \\
(\mu \mathrm{m})\end{array}$ & $\begin{array}{c}\text { mean } \\
\hat{\gamma}(\mu \mathrm{m})\end{array}$ & $\begin{array}{c}\text { mode } \\
(\mu \mathrm{m})\end{array}$ \\
\hline \hline none & 0.68 & 0.60 & 0.57 & 0.54 \\
min,max & 0.59 & 0.54 & 0.49 & 0.47 \\
corr,min,max & 0.73 & 0.61 & 0.50 & 0.47 \\
corr. & 0.81 & 0.74 & 0.55 & 0.51 \\
\hline
\end{tabular}

Table 5: Overall means and modes for the different candidate fibre selection techniques. The mean of $\hat{\gamma}$ was calculated with (17). The mode was calculated using kernel density estimation.

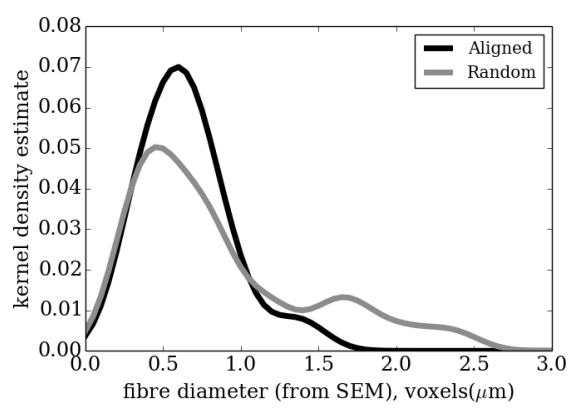

Figure 14: Kernel Density Estimates (KDE) for the fibre diameter estimates measured from the high resolution SEM images for an aligned and a random fibre sample.

The diameter estimates using the 3D data appear to agree well with diameter estimates that have been obtained from Scanning Electron Microscope (SEM) images of the same samples. Six SEM images for each fibre sample were used, three from the front and three from the back of the fibre samples were acquired. Exemplar images are shown earlier in Fig. 8. These images were used to manually measure the widths of the fibres at 20 semi-randomly locations for each image, producing 120 measurements for each fibre sample. Kernel Density Estimation (KDE) using a Gaussian kernel was then used to estimate the mean and mode of these diameter estimates. This process was performed for a range of different kernel bandwidths as can be seen from the estimates shown in Table 6. Exemplar KDE estimated densities for a bandwidth of $0.15 \mu \mathrm{m}$ can be seen in Fig. 14 .

The SEM based measurements demonstrate reasonably good agreement with the XCT based measurements. For example, consider the SEM mode estimates for a bandwidth of $0.15 \mu \mathrm{m}$, resulting in a diameter estimate for the aligned fibres of $0.60 \mu \mathrm{m}$ and a diameter estimate of $0.45 \mu \mathrm{m}$ for the random fibre sample. This corresponds very well with the modal fibre estimate using the XCT data of $0.61 \mu \mathrm{m}$ and $0.47 \mu \mathrm{m}$ for the aligned and random fibre samples respectively, representing, at most a $4 \%$ error. The SEM results also appear to show a very long tail in diameters of the randomly distributed fibre samples, which explains the large variability seen in the XCT diameter estimates. The SEM measurements can be considered a gold standard due to the high resolution of these images in comparison to the XCT. An advantage of the XCT over SEM, however, is that $\mathrm{XCT}$ is able to provide information relating to the relation of the fibres in $3 \mathrm{D}$, such as the $3 \mathrm{D}$ fibre orientation distribution which is considered next.

\section{Orientation Distribution Results}

A comparison of the effects of the different candidate fibre selection criteria on the orientation distribution can be seen in Figs. 15 and 16.

The orientation distribution can also be conveniently summarised with the multiscale entropy using (18), as can be seen in Fig. 17. 


\begin{tabular}{|c|c|c|c|c|}
\hline $\begin{array}{c}\text { KDE } \\
\text { bandwidth } \\
(\mu \mathrm{m})\end{array}$ & \multicolumn{2}{|c|}{ Aligned } & \multicolumn{2}{c|}{ Random } \\
$(\mu \mathrm{m})$ & $\begin{array}{c}\text { mode } \\
(\mu \mathrm{m})\end{array}$ & $\begin{array}{c}\text { mean } \\
(\mu \mathrm{m})\end{array}$ & $\begin{array}{c}\text { mode } \\
(\mu \mathrm{m})\end{array}$ \\
\hline \hline 0.05 & 0.65 & 0.60 & 0.87 & 0.45 \\
0.10 & 0.65 & 0.60 & 0.87 & 0.40 \\
0.15 & 0.65 & 0.60 & 0.87 & 0.45 \\
0.20 & 0.66 & 0.60 & 0.88 & 0.50 \\
0.25 & 0.67 & 0.60 & 0.89 & 0.55 \\
0.30 & 0.68 & 0.60 & 0.91 & 0.55 \\
0.35 & 0.70 & 0.60 & 0.93 & 0.55 \\
0.40 & 0.72 & 0.60 & 0.95 & 0.60 \\
0.45 & 0.75 & 0.60 & 0.97 & 0.60 \\
0.50 & 0.77 & 0.60 & 0.99 & 0.60 \\
\hline
\end{tabular}

Table 6: Means and modes estimated using SEM image based measurements together with Kernel Density Estimation (KDE) across a range of bandwidths. The mode, in particular, demonstrates very good agreement with the 3D fibre diameter estimates obtained using the correlation and minimum and maximum checks.
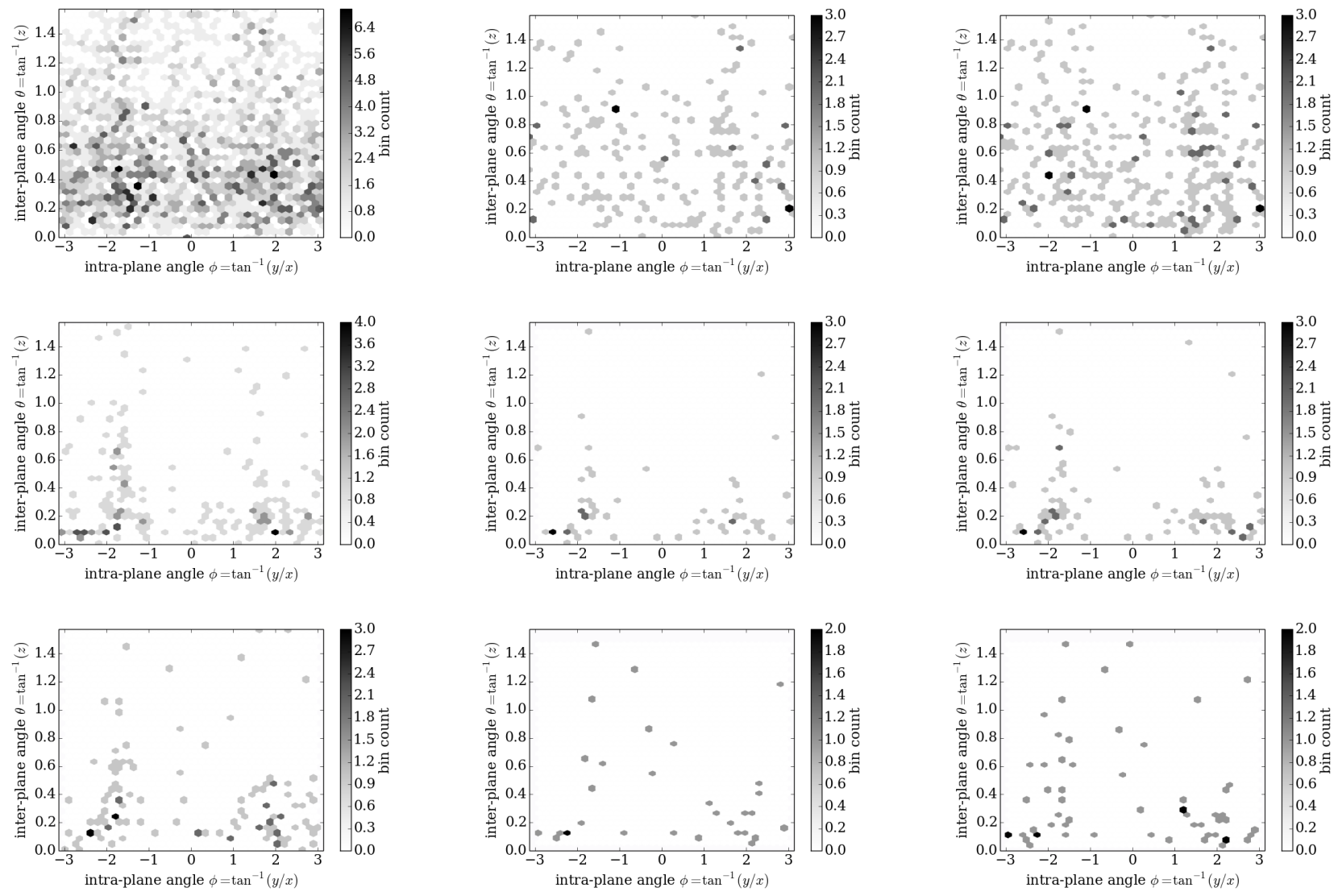

Figure 15: Comparison of orientation distributions, for a sample with aligned fibres, across a number of scales $(\eta \in(0.15,0.50,0.75))$ for the different selection criteria. Left column: selection using minimums and maximums of line profiles, i.e. (8) \& (9); middle column: selection using the minimums and maximums of line profiles (8), (9) and the correlation between line profiles i.e. (10); right column: selection using just correlation, (10). The orientation distributions using correlation appear to be the most effective at reducing the number of outliers here. 

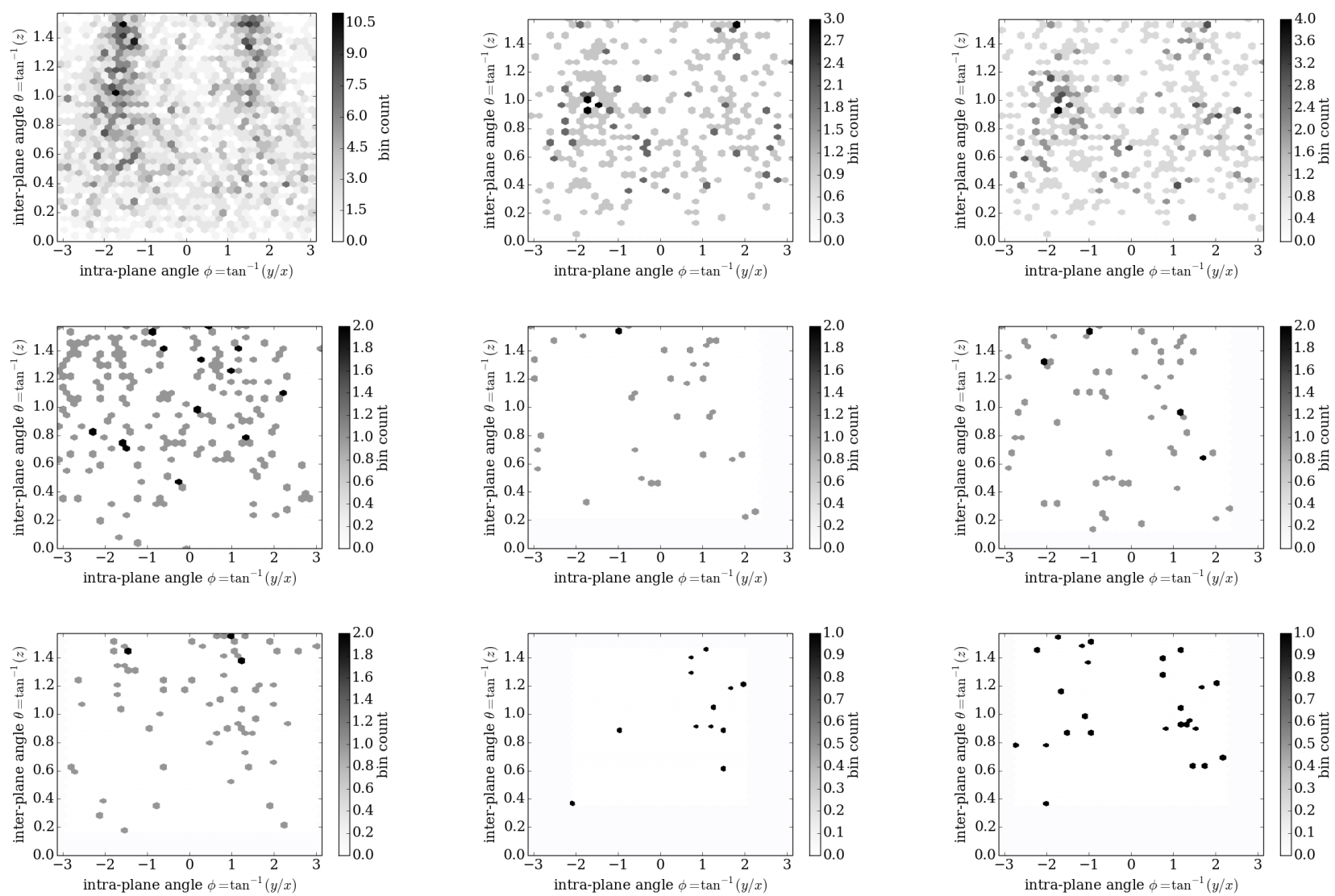

Figure 16: Comparison of orientation distributions, for a sample with randomly distributed fibres, across a number of scales $(\eta \in(0.15,0.50,0.75))$ for the different selection criteria. Left column: selection using minimums and maximums of line profiles, i.e. (8) \& (9); middle column: selection using the minimums and maximums of line profiles (8), (9) and the correlation between line profiles i.e. (10); right column: selection using just correlation, (10). The orientation distributions using correlation appear to be the most effective at reducing the number of outliers here.
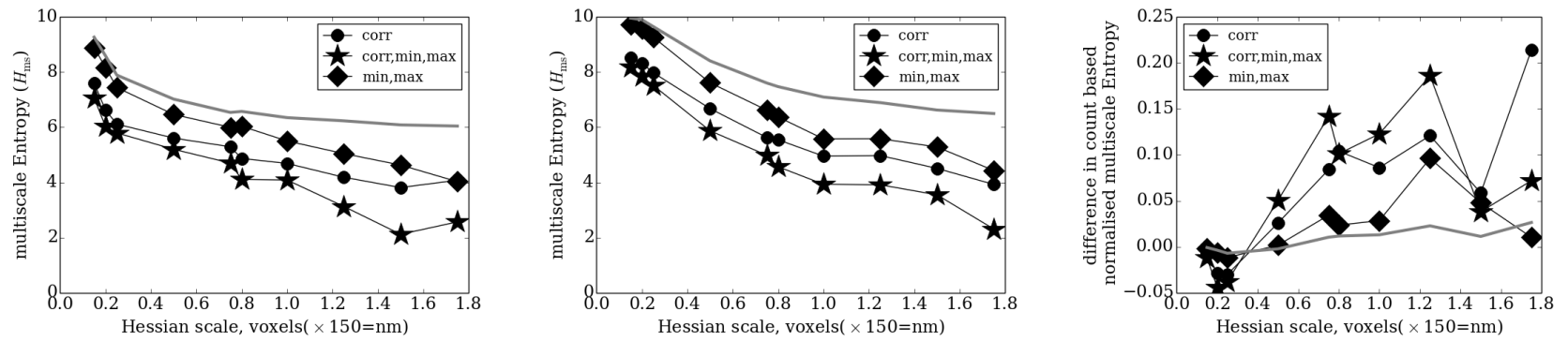

Figure 17: Multiscale Entropy calculations as a function of the Hessian scale and the candidate fibre selection techniques for a sample with aligned fibres (left) and one with randomly distributed fibres (middle). On the (right) is $\zeta_{\eta}$, from (19) which is the difference between the random and aligned multiscale entropies after normalization, based on the number of detected fibres for the different schemes. Positive values indicate the random fibre sample resulted in a higher Entropy whilst negative indicate the aligned fibres resulted in a higher Entropy. 


\begin{tabular}{|c|c|c|c|c|}
\hline selection & mean & st.dev. & max. & min. \\
\hline \hline none & 0.01 & 0.01 & 0.03 & -0.01 \\
\hline max. \& min. (8), (9) & 0.02 & 0.03 & 0.10 & -0.01 \\
\hline max. \& min. \& corr. (8), (9), (10) & 0.06 & 0.07 & 0.19 & -0.04 \\
\hline corr. (10) & 0.06 & 0.07 & 0.21 & -0.03 \\
\hline
\end{tabular}

Table 7: Summary statistics across scale for the differences in the multiscale entropy for each of the fibre selection processes. All units are in $\mu \mathrm{m}$.

The results in Fig. 17 appear to show a reduction in the entropy with increasing scale in the Hessian which could be due to the reduction in the number of detected fibres. This is somewhat confirmed by the lower entropy estimates for the correlation combined with the min. and max. candidate selection process which has the fewest detections overall, see e.g. Table 3.

To take account of the apparent dependence of the multiscale entropy on the number of detections, the multiscale entropy estimates were normalised by the number of detected fibres for each different candidate fibre selection scheme. Following the normalisation, the difference between the normalised multiscale Entropies was calculated i.e.

$$
\zeta_{\eta}=\frac{H_{\mathrm{ms}, \eta}^{\mathrm{random}}}{\beta_{\eta}^{\mathrm{random}}}-\frac{H_{\mathrm{ms}, \eta}^{\text {aligned }}}{\beta_{\eta}^{\text {aligned }}}
$$

The difference in the multiscale Entropies as a function of scale are generally greater for the random fibre sample. This can be seen with the summary statistics across scale in Table 7.

It is interesting to note that there is a sharp drop in the difference of the entropies for very small scales $(\eta<0.5)$ and also for the larger scale values $(\eta>1.5)$. This can be compared with visual observation of the fibres in the SEM images in Fig. 8. The thinner and the thicker fibres for the aligned sample are less well aligned than the medium thickness fibres.

\section{Conclusions}

This work has described the development and testing of a nano-scale fibre detection process for the detection of fibres in XCT of PCL electrospun based material samples. Fibres are detected across a range of scales and are further selected based on properties suitable for detailed measurements. The measurements obtained include the diameters of the fibres and also the orientation to form an orientation distribution for a particular fibre sample. The correctness of the obtained measurements has been validated by measurements manually determined from SEM based images. The obtained orientation distributions were further summarised using a multiscale entropy descriptor which was found to provide higher entropy across a range of scales for the randomised fibre sample.

\section{Acknowledgments}

The authors would like to acknowledge the generous support of the XCT facilities at the University of Portsmouth's Zeiss Global Centre and the SEM facilities in the University of Portsmouth's Institute of Biomedical and Biomolecular Science. The authors of the work presented here would also like to thank the many authors of ITK Johnson et al. (2013) and VTK Schroeder et al. (2006). 


\section{References}

Ahmed, S., Klassen, T., Keyes, S., Daly, M., Jones, D., Mavrogordato, M., Sinclair, I., and Roose, T. (2016). Imaging the interaction of roots and phosphate fertiliser granules using 4D X-ray tomography. Plant Soil.

Al-Attabi, R., Dume, L. F., Kong, L., Schtz, J. A., and Morsi, Y. (2018). High efficiency poly(acrylonitrile) electrospun nanofiber membranes for airborne nanomaterials filtration. Advanced Engineering Materials, 20(1):1700572-n/a.

Axelsson, M. (2008). Estimating 3D fibre orientation in volume images. In Pattern Recognition, 2008. ICPR 2008. 19th International Conference on, pages 1-4.

Axelsson, M. and Svensson, S. (2010). 3D pore structure characterisation of paper. Pattern Anal. Applic., 13:159-172.

Barrett, J. F. and Keat, N. (2004). Artifacts in CT: Recognition and avoidance. RadioGraphics, 24(6):1679-1691.

Bresenham, J. (1965). Algorithm for computer control of a digital plotter. IBM Systems J., 4(1):2530.

Cheng, H., Chawla, A., Yang, Y., Li, Y., Zhang, J., Jang, H. L., and Khademhosseini, A. (2017). Development of nanomaterials for bone-targeted drug delivery. Drug Discovery Today, 22(9):1336 - 1350. SI:Therapeutic Nanomaterials.

Chiverton, J., Ige, O., Barnett, S., and Parry, T. (2017). Multiscale shannon's entropy modelling of orientation and distance in steel fiber micro-tomography data. IEEE Trans. Image Processing. Accepted.

Chiverton, J. and Wells, K. (2008). Adaptive partial volume classification of MRI data. Physics Med. Biol., 53(20):5577.

Deravi, L. F., Sinatra, N. R., Chantre, C. O., Nesmith, A. P., Yuan, H., Deravi, S. K., Goss, J. A., MacQueen, L. A., Badrossamy, M. R., Gonzalez, G. M., Phillips, M. D., and Parker, K. K. (2017). Design and fabrication of fibrous nanomaterials using pull spinning. Macromolecular Materials and Engineering, 302(3):1600404-n/a. 1600404.

Drechsler, K. and Laura, C. O. (2010). Comparison of vesselness functions for multiscale analysis of the liver vasculature. In Proc. 10th IEEE Int. Conf. Info. Tech. Appls. in Biomedicine, pages 1-5.

Emerson, M. J., Jespersen, K. M., Dahl, A. B., Conradsen, K., and Mikkelsen, L. P. (2017). Individual fibre segmentation from $3 \mathrm{~d} x$-ray computed tomography for characterising the fibre orientation in unidirectional composite materials. Composites Part A: Applied Science and Manufacturing, 97:83 - 92.

Frangi, A. F., Niessen, W. J., Vincken, K. L., and Viergever, M. A. (1998). Multiscale vessel enhancement filtering. In Wells, W. M., Colchester, A., and Delp, S., editors, Medical Image Computing and Computer-Assisted Intervention - MICCAI'98, pages 130-137, Berlin, Heidelberg. Springer Berlin Heidelberg.

Hassiba, A., El-Zowalaty, M., Webster, T., Abdullah, A., Nasrallah, G., Khalil, K., Luyt, A., and Elzatahry, A. (2017). Synthesis, characterization, and antimicrobial properties of novel double layer nanocomposite electrospun fibers for wound dressing applications. Int. J. Nanomedicine, 12:2205-2213. 
Hotaling, N., Bharti, K., Kriel, H., and Simon, C. (2015). Diameterj: A validated open source nanofiber diameter measurement tool. Biomaterials, 61:327 - 338.

Huang, X., Wen, D., Zhao, Y., Wang, Q., Zhou, W., and Deng, D. (2016). Skeleton-based tracing of curved fibers from 3D X-ray microtomographic imaging. Results in Physics, 6:170 - 177.

Hunter, J. D. (2007). Matplotlib: A 2d graphics environment. Computing In Science \& Engineering, 9(3):90-95.

Jiang, S., Song, P., Guo, H., Zhang, X., Ren, Y., Liu, H., Song, X., and Kong, M. (2017). Blending $\mathrm{plla} /$ tannin-grafted pcl fiber membrane for skin tissue engineering. Journal of Materials Science, 52(3):1617-1624.

Jimenez-Carretero, D., Santos, A., Kerkstra, S., Rudyanto, R. D., and Ledesma-Carbayo, M. J. (2013). 3D Frangi-based lung vessel enhancement filter penalizing airways. In 2013 IEEE 10th International Symposium on Biomedical Imaging, pages 926-929.

Johnson, H. J., McCormick, M., Ibáñez, L., and Consortium, T. I. S. (2013). The ITK Software Guide. Kitware, Inc., third edition.

Kitrungrotsakul, T., Han, X.-H., Iwamoto, Y., Foruzan, A., Lin, L., and Chen, Y.-W. (2017). Robust hepatic vessel segmentation using multi deep convolution network. volume 10137, pages 1013711-1013711-6.

Maksimcuka, J., Obata, A., Sampson, W. W., Blanc, R., Gao, C., Withers, P. J., Tsigkou, O., Kasuga, T., Lee, P. D., and Poologasundarampillai, G. (2017). X-ray tomographic imaging of tensile deformation modes of electrospun biodegradable polyester fibers. Frontiers in Materials, $4: 43$.

Mansfield, J., Yu, J., Attenburrow, D., Moger, J., Tirlapur, U., Urban, J., Cui, Z., and Winlove, P. (2009). The elastin network: its relationship with collagen and cells in articular cartilage as visualized by multiphoton microscopy. Journal of Anatomy, 215(6):682-691.

Mets, O., de Jong, P., van Ginneken, B., Gietema, H., and Lammers, J. (2012). Quantitative computed tomography in COPD: Possibilities and limitations. Lung, 190:133-145.

Mostao-Guidolin, L., Rosin, N. L., and Hackett, T.-L. (2017). Imaging collagen in scar tissue: Developments in second harmonic generation microscopy for biomedical applications. International Journal of Molecular Sciences, 18(8).

Pasinszki, T., Krebsz, M., Tung, T. T., and Losic, D. (2017). Carbon nanomaterial based biosensors for non-invasive detection of cancer and disease biomarkers for clinical diagnosis. Sensors, 17(8).

Rigort, A., Gnther, D., Hegerl, R., Baum, D., Weber, B., Prohaska, S., Medalia, O., Baumeister, W., and Hege, H.-C. (2012). Automated segmentation of electron tomograms for a quantitative description of actin filament networks. Journal of Structural Biology, 177(1):135 - 144.

Rudyanto, R., Kerkstra, S., van Rikxoort, E., et al. (2014). Comparing algorithms for automated vessel segmentation in computed tomography scans of the lung: the VESSEL12 study. Medical Image Analysis, 18(7):1217 - 1232.

Sato, Y., Nakajima, S., Shiraga, N., et al. (1998). 3D multi-scale line filter for segmentation and visualization of curvilinear structures in medical images. Med. Image Anal., 2(2):143-168.

Schroeder, W., Martin, K., and Lorensen, B. (2006). The Visualization Toolkit. Kitware, Inc., fourth edition. 
Sensini, A., Gualandi, C., Cristofolini, L., Tozzi, G., Dicarlo, M., Teti, G., Mattioli-Belmonte, M., and Focarete, M. L. (2017). Biofabrication of bundles of poly(lactic acid)-collagen blends mimicking the fascicles of the human Achille tendon. Biofabrication, 9(1):015025.

Sims, A. J., Bennett, M. K., and Murray, A. (2002). Comparison of semi-automated image analysis and manual methods for tissue quantification in pancreatic carcinoma. Physics Med. Biol., 47(8):1255.

Weber, B., Greenan, G., Prohaska, S., Baum, D., Hege, H.-C., Mller-Reichert, T., Hyman, A. A., and Verbavatz, J.-M. (2012). Automated tracing of microtubules in electron tomograms of plastic embedded samples of caenorhabditis elegans embryos. Journal of Structural Biology, 178(2):129 - 138. Special Issue: Electron Tomography.

Yau, W. W. Y., Long, H., Gauthier, N. C., Chan, J. K. Y., and Chew, S. Y. (2015). The effects of nanofiber diameter and orientation on sirna uptake and gene silencing. Biomaterials, 37:94 106. 